\title{
O Impacto dos usos Interativo e Diagnóstico dos Orçamentos sobre a Eficácia das Equipes: Retornando ao Básico
}

Gabriel Corrêa Teles Assis
Mestrado em Ciências Contábeis pela Universidade Federal do Rio de Janeiro - UFRJ
Contador

Avenida Pasteur, 250, sala 251, Urca, Rio de Janeiro/RJ. CEP: 22290-250

E-mail: gabriel.tassis@gmail.com

Moacir Sancovschi

Doutorado em Administração pelo Instituto Coppead de Administração

Professor da Universidade Federal do Rio de Janeiro - UFRJ Avenida Pasteur, 250, sala 251, Urca, Rio de Janeiro/RJ. CEP: 22290-250

E-mail:msancov@facc.ufrj.br

José Francisco Rezende Doutorado em Administração pelo Instituto Coppead de Administração

Pesquisador associado OG Observatório Gerencial Membro da Comissão Especial de Governança Corporativa do CRA-RJ Rua Professor Gabizo, 197. Tijuca. Rio de Janeiro/RJ. CEP: 20271-064

E-mail: rezende.jf@gmail.com

\section{RESUMO}

Esta pesquisa tem como objetivo reafirmar que, nas unidades organizacionais onde a incerteza da tarefa é baixa, os orçamentos são usados diagnosticamente e, nas unidades onde a incerteza da tarefa é alta, os orçamentos são usados interativamente; e que a adoção dos estilos de uso dos orçamentos adequados ao nível de incerteza da tarefa contribui para a eficácia coletiva percebida em equipes formais de trabalho e para a eficácia destas equipes. Através de um estudo de caso, foram entrevistados representantes da Coordenação de Geodésia e da Supervisão de Gravimetria, duas unidades do IBGE que desempenham atividades com níveis de incerteza distintos. Conforme esperado, observou-se que, na Coordenação de Geodésia, que realiza tarefas com elevado nível de incerteza, o orçamento é usado de forma interativa e há a percepção de que a eficácia coletiva e a eficácia da equipe são satisfatórias. $\mathrm{Na}$ Supervisão de Gravimetria, que desempenha tarefas com baixo nível de incerteza, o orçamento é usado de forma diagnóstica e há a percepção de que a eficácia coletiva e a eficácia da equipe também são satisfatórias. A principal contribuição deste trabalho é reiterar que a análise e o projeto de sistemas de controle não podem prescindir de uma avaliação do nível de incerteza das tarefas onde eles são ou serão adotados. 
O Impacto dos usos Interativo e Diagnóstico dos Orçamentos sobre a Eficácia das Equipes:

Retornando ao Básico

Gabriel Corrêa Teles Assis, Moacir Sancovschi, José Francisco Rezende

Palavras-chave: Orçamentos operacionais. Sistemas interativos. Sistemas diagnósticos. Incerteza da Tarefa.

The Impact of Interactive and Diagnostic uses of Budgets on Team Effectiveness: Back to the Basics

\section{ABSTRACT}

This research has the objective to reaffirm that, in organizational units where the task uncertainty is low, budgets are used diagnostically, and, in units where task uncertainty is high, budgets are used interactively; and that the adoption budget use styles appropriate to the task's level of uncertainty contributes to the perceived collective effectiveness in formal work teams and to these teams' effectiveness. Through a case study, representatives of the Coordination of Geodesy and the Supervision of Gravimetry, two IBGE units that perform activities with different levels of uncertainty, were interviewed. As expected, it was observed that, in the Coordination of Geodesy, which performs tasks with a high level of uncertainty, the budget is used interactively and there is a perception that the collective effectiveness and the team effectiveness are satisfactory. In Gravimetry Supervision, which performs tasks with a low level of uncertainty, the budget is used in a diagnostic manner and there is a perception that the collective effectiveness and the team effectiveness are also satisfactory. The main contribution of this work is to reiterate that the analysis and the design of control systems cannot be done without an assessment of the level of uncertainty of the tasks where these systems are or will be adopted.

Keywords: Operational budgets. Interactive systems. Diagnostic systems. Task uncertainty.

\section{El Impacto de los usos Interactivo y de Diagnóstico de los Presupuestos en la Eficacia del Equipo: De vuelta a lo Básico}

\section{RESUMEN}

Esta investigación tiene como objetivo reafirmar que, en las unidades organizativas donde la incertidumbre de la tarea es baja, los presupuestos se utilizan de forma diagnóstica $y$, en las unidades donde la incertidumbre de la tarea es alta, los presupuestos se utilizan de forma interactiva; y que la adopción de estilos de uso de presupuestos adecuados al nivel de incertidumbre de la tarea contribuye a la efectividad colectiva percibida en los equipos de trabajo formales y a la efectividad de estos equipos. A través de un estudio de caso, se entrevistó a representantes de la Coordinación de Geodesia y de la Supervisión de Gravimetría, dos unidades del IBGE 
que realizan actividades con diferentes niveles de incertidumbre. Como era de esperar, se observó que, en la Coordinación de Geodesia, que realiza tareas con un alto nivel de incertidumbre, el presupuesto se utiliza de forma interactiva y existe la percepción de que la efectividad colectiva y de equipo es satisfactoria. En la Supervisión de Gravimetría, que realiza tareas con un bajo nivel de incertidumbre, el presupuesto se utiliza de manera diagnóstica y existe la percepción de que la efectividad colectiva y de equipo también es satisfactoria. El principal aporte de este trabajo es reiterar que el análisis y diseño de sistemas de control no puede prescindir de una valoración del nivel de incertidumbre de las tareas donde se encuentran o serán adoptados.

Palabras clave: Presupuestos operacionales. Sistemas interactivos. Sistemas diagnósticos. Incertidumbre de la tarea.

\section{INTRODUÇÃO}

Os livros de contabilidade de custos e de contabilidade gerencial normalmente ensinam como elaborar orçamentos (Atkinson et al., 2015; Garrison et al., 2013; Horngren et al., 2015). No entanto, Merchant e Van Der Stede (2012) argumentam que os principais benefícios dos orçamentos advêm do processo de prepará-los, e da forma como eles são usados na administração das organizações, tenham elas fins lucrativos ou não.

As consequências dos estilos com que os orçamentos são usados na administração têm sido estudadas há bastante tempo (Hopwood, 1972; Otley, 1978). As primeiras pesquisas basearam-se em conceitos e modelos concebidos e empregados por professores de administração e de sociologia (Perrow, 1967; Ouchi, 1979), mas à medida que professores de contabilidade foram desenvolvendo modelos para a análise e projeto de sistemas de controle nas organizações (Merchant, 1985; Simons, 1995), esses modelos passaram a ser adotados para estudar as implicações dos estilos com que os orçamentos são usados por administradores.

Tais estudos têm fornecido evidências consistentes de que a incerteza da tarefa é um marco relevante para o projeto de sistemas de controle em geral, e para a escolha de um estilo de uso dos orçamentos em particular. Contudo, há de se reconhecer que esses estudos vem sendo vítimas da "tirania das palavras" - o uso de diferentes 
O Impacto dos usos Interativo e Diagnóstico dos Orçamentos sobre a Eficácia das Equipes:

Retornando ao Básico

Gabriel Corrêa Teles Assis, Moacir Sancovschi, José Francisco Rezende

nomes para expressar o mesmo conceito e o uso do mesmo nome para expressar conceitos distintos (Samuelson, 1973, p. 10); e da crescente tendência que membros da comunidade acadêmica têm de rejeitar livros e pesquisas com base no critério do tempo decorrido da publicação, independente do mérito que tenham.

Este trabalho tem como objetivos reafirmar, com base em um estudo de caso, que, nas unidades das organizações onde a incerteza da tarefa é baixa, os orçamentos são usados de forma diagnóstica; e, nas unidades onde a incerteza da tarefa é alta, os orçamentos são usados de forma interativa; e que a adoção de estilos de uso dos orçamentos adequados ao nível de incerteza da tarefa contribui para a eficácia coletiva percebida em equipes formais de trabalho e para a eficácia destas equipes. Ele foi concebido a partir de uma crítica à duas das hipóteses que Chong e Mahama (2014) propuseram, mas que não nao estavam alinhadas ao modelo de controle de Simons (1995) que fundamentou a pesquisa que fizeram, nem estavam alinhadas com os resultados de estudos empíricos sobre sistemas de contabilidade e controle nas organizações.

As hipóteses de Chong e Mahama (2014) assumiram que os orçamentos poderiam ser usados concomitantemente de forma diagnóstica e interativa, e dessa forma contribuiriam para a eficácia coletiva percebida em equipes formais de trabalho e para a eficácia destas equipes. Chong e Mahama (2014), diante das evidências que reuniram, abandonaram os pressupostos da pesquisa, sem reconhecer que suas hipóteses não procediam.

São duas as principais contribuições deste trabalho. A primeira é que ele fornece subsídios para esclarecer mais uma vez que o insucesso de Chong e Mahama (2014) em encontrar relações positivas e significativas entre o uso diagnóstico do orçamento e a eficácia coletiva percebida e a eficácia das equipes deveu-se ao fato de que eles entrevistaram funcionários dos departamentos de pesquisa de empresas de biotecnologia, unidades onde as atividades não podem ser previamente planejadas. $O$ uso interativo do orçamento nestes departamentos é parte de um processo de experimentação necessário para equilibrar a necessidade de buscar respostas para 
O Impacto dos usos Interativo e Diagnóstico dos Orçamentos sobre a Eficácia das Equipes:

Retornando ao Básico

Gabriel Corrêa Teles Assis, Moacir Sancovschi, José Francisco Rezende

desafios técnicos, considerando as restrições impostas pelos recursos disponíveis e pela necessidade de satisfazer as demandas dos investidores.

A segunda contribuição deste trabalho é que o caso que ele descreve respalda, mais uma vez, a orientação constante nos livros de contabilidade e controle gerencial de que a análise e o projeto de sistemas de controle não podem prescindir de considerar a incerteza das atividades realizadas nas diferentes unidades que integram as organizações, independente das suas finalidades.

São quatro, as seções que completam este artigo. A seção 2 apresenta a revisão de literatura e o modelo desta pesquisa. O capítulo 3 expõe a metodologia de pesquisa adotada e as limitações que ela impõe às análises feitas. O capítulo 4 relata e analisa o caso estudado. E para encerrar, o capítulo 5 apresenta as considerações finais.

\section{REVISÃO DE LITERATURA}

\subsection{Estilos de Uso dos Orçamentos, Eficácia Coletiva Percebida em Equipes e Eficácia das Equipes.}

Chong e Mahama (2014) estudaram o impacto dos estilos com que os orçamentos são usados nas unidades de pesquisa de empresas de biotecnologia sobre a eficácia coletiva percebida em equipes formais de trabalho, e sobre a eficácia dessas equipes. Eles consideraram dois possíveis estilos de uso dos orçamentos uso diagnóstico e uso interativo.

Segundo Simons (1995), o uso diagnóstico dos orçamentos pressupõe que eles expressem planos que devem ser realizados e objetivos que devem ser alcançados. Sendo assim, os relatórios orçamentários são empregados pelos gerentes para avaliar se os planos estão sendo concretizados, se os objetivos estão sendo atingidos, e, caso haja problemas, para tomar as providências cabíveis para corrigi-los. Nessa concepção o controle orçamentário se baseia no modelo de controle cibernético ou de feedback negativo (Hofstede, 1981). 
O Impacto dos usos Interativo e Diagnóstico dos Orçamentos sobre a Eficácia das Equipes:

Retornando ao Básico

Gabriel Corrêa Teles Assis, Moacir Sancovschi, José Francisco Rezende

O uso interativo dos orçamentos, de acordo com Simons (1995), pressupõe que a finalidade dos orçamentos seja "promover consistência nas ações desempenhadas pelas organizações, sejam elas intencionais ou não" (Mintzberg, 1987). Para isso, os relatórios orçamentários são considerados produtos de sistemas formais que promovem o compartilhamento de informações entre gerentes e funcionários das organizações e estimulam a criatividade.

A eficácia coletiva percebida em equipes de trabalho foi definida na referida pesquisa como "as crenças dos membros das equipes nas suas habilidades coletivas de organizar e de executar as tarefas que Ihes são delegadas" (Chong \& Mahama, 2014).

A eficácia das equipes foi avaliada por Chong e Mahama (2014) por meio das percepções dos membros das equipes acerca (1) da qualidade, da precisão e da quantidade do trabalho realizado; (2) da pontualidade e da satisfação com o trabalho feito; e (3) da eficiência com que as operações foram realizadas.

Os resultados alcançados por Chong e Mahama (2014) suportaram as hipóteses de que há uma relação direta e positiva entre o uso interativo dos orçamentos e a eficácia das equipes, e entre o uso interativo do orçamento e a eficácia coletiva percebida em equipes. Eles também reuniram evidências de que a relação entre o uso interativo dos orçamentos e a eficácia das equipes foi parcialmente mediada pela eficácia coletiva percebida das equipes.

Apesar de terem previsto que deveria haver relações diretas e positivas entre o uso diagnóstico dos orçamentos, a eficácia coletiva percebida em equipes e a eficácia das equipes, os resultados obtidos por Chong e Mahama (2014) para essas relações foram negativos e não significantes. Eles, então, abandonaram as hipóteses que propuseram, e atribuíram esses resultados ao fato de que os entrevistados trabalhavam em unidades de pesquisa onde predominam atividades criativas e inovadoras, e que resultados similares foram obtidos em pesquisas anteriores. 
O Impacto dos usos Interativo e Diagnóstico dos Orçamentos sobre a Eficácia das Equipes:

Retornando ao Básico

Gabriel Corrêa Teles Assis, Moacir Sancovschi, José Francisco Rezende

\subsection{Expectativas Injustificadas de Chong e Mahama}

As suposições de Chong e Mahama (2014) de que existiriam relações diretas e positivas entre o uso diagnóstico dos orçamentos nos departamentos de pesquisa de empresas de biotecnologia e a eficácia coletiva percebida em equipes, e a eficácia das equipes não encontram respaldo no modelo das alavancas de controle de Simons (1995).

O Quadro 1 reproduz as condições que Simons (1995) considera necessárias e suficientes para o uso dos controles diagnósticos e dos controles interativos.

\begin{tabular}{|c|c|c|}
\hline & Sistemas de Controle Diagnósticos & Sistemas de Controles Interativos \\
\hline $\begin{array}{l}\text { Condições } \\
\text { para usar }\end{array}$ & $\begin{array}{l}\text { Padrões de desempenho podem ser } \\
\text { preestabelecidos; resultados podem ser } \\
\text { medidos; informações de feedback podem ser } \\
\text { usadas para influenciar ou corrigir os desvios } \\
\text { dos padrões; o processo ou os resultados são } \\
\text { variáveis críticas de desempenho. }\end{array}$ & $\begin{array}{l}\text { Incertezas estratégicas requerem a } \\
\text { busca frequente por mudanças de } \\
\text { ruptura e de oportunidades. }\end{array}$ \\
\hline
\end{tabular}

Quadro 1. Sistemas de Controle Diagnósticos vs Sistemas de Controle Interativos Fonte: Simons (1995)

Comparando as condições para o uso adequado dos dois sistemas de controle, fica evidente que elas não podem coexistir em uma atividade. É bastante improvável que uma atividade para a qual (i) padrões podem ser previamente estabelecidos; (ii) seus resultados podem ser medidos e comparados com os padrões; (iii) as informações obtidas nessas comparações podem ser usadas para corrigir os desvios em relação aos planos; e (iv) garantir que o processo seja realizado sem surpresas e alcançar os resultados almejados seja crítico para o seu sucesso, apresente incertezas estratégicas que requerem a busca frequente de mudanças radicais e de novas oportunidades.

Para reforçar esta constatação nada melhor do que dar voz ao próprio Simons (1995). Ele afirma textualmente que "controles diagnósticos são difíceis de implementar se existir um alto grau de novidade no processo a ser controlado. Por essa razão, controles diagnósticos válidos são notoriamente difíceis de implementar em laboratórios de pesquisa e desenvolvimento". Um pouco mais adiante, Simons (1995) complementa, 
O Impacto dos usos Interativo e Diagnóstico dos Orçamentos sobre a Eficácia das Equipes:

Retornando ao Básico

Gabriel Corrêa Teles Assis, Moacir Sancovschi, José Francisco Rezende

apontando que "a habilidade de corrigir os desvios [típica das atividades rotineiras] sugere que os controles diagnósticos são apropriados somente para processos nos quais os participantes têm influência significativa".

Há de se reconhecer que as condições consideradas por Simons (1995) como necessárias e suficientes para empregar controles diagnósticos já são conhecidas e referenciadas há bastante tempo. Hofstede (1981), por exemplo, as apresenta da seguinte forma: (i) Deve haver um padrão que expresse o alcance eficiente e eficaz dos objetivos da organização; (ii) Deve haver meios de se medir a realização eficiente e eficaz dos objetivos; (iii) Deve haver um repertório de intervenções que possa ser usado para corrigir os desvios observados entre o desempenho obtido e o padrão requerido; e (iv) A atividade deve ser repetitiva.

É necessário ressalvar que sistemas de controle diagnóstico somente podem e devem ser utilizados no âmbito das unidades de pesquisa de empresas de biotecnologia para controlar as partes rotineiras das suas atividades. Esse é o caso da utilização e do descarte de materiais empregados nos experimentos, da segurança que deve preservada no ambiente de trabalho, da preparação dos relatórios de pesquisa etc.

\subsection{Sistemas de Controle e Incerteza da Tarefa}

A concepção de que a incerteza da tarefa é uma referência relevante para o projeto e para a avaliação dos sistemas de controle tem sido expressa e reafirmada por diversos professores ao longo do tempo. Anthony (1974) a destaca quando contrapõe o controle gerencial ao controle técnico. Dermer (1977) a utiliza quando classifica os processos num contínuo onde os processos estruturados estão em um extremo, e no outro, estão os processos desestruturados; e mostra que há diferenças entre o planejamento e o controle destes processos. Landau e Stout (1979) recorrem à incerteza da tarefa para criticar a crença de que "administrar é controlar". Seijts e Latham (2012) a empregam para recomendar as condições em que se devem 
O Impacto dos usos Interativo e Diagnóstico dos Orçamentos sobre a Eficácia das Equipes:

Retornando ao Básico

Gabriel Corrêa Teles Assis, Moacir Sancovschi, José Francisco Rezende

estabelecer metas de desempenho (resultados esperados) e que se devem fixar metas de aprendizado para as atividades.

\subsubsection{Resultados de Pesquisas Empíricas}

Van de Ven, Delbecq e Koenig (1976) entrevistaram funcionários de departamentos de uma agência de segurança do trabalho, e reuniram informações que, em geral, suportaram a hipótese de que "aumentos no grau de incerteza da tarefa para uma unidade organizacional estão associados (i) ao menor uso de modos de coordenação impessoal (programas, regras, planos, etc.), (ii) ao maior uso de modos de coordenação pessoal (uso de canais verticais e horizontais de comunicação), e (iii) ao uso substancialmente maior de modos de coordenação grupal (reuniões agendadas e não agendadas)".

A incerteza da tarefa, de acordo com estes pesquisadores, refere-se à variabilidade e à dificuldade do trabalho realizado em uma unidade organizacional, tal como definidas por Perrow (1967). A variabilidade da tarefa é determinada pelo número de casos excepcionais encontrados no trabalho. Ela pode ser baixa ou alta. $E$ a dificuldade da tarefa é determinada pela natureza do processo de busca realizado quando ocorre um caso excepcional no desempenho da atividade. Em um extremo estão buscas lógicas baseadas em análise (dificuldade baixa), e no outro, estão buscas baseadas na experiência acumulada, na intuição, em conjecturas, em apostas, etc (dificuldade alta).

Daft e Macintosh (1981) entrevistaram os funcionários de departamentos de diferentes organizações e observaram que (i) a quantidade de informações usada nos departamentos cresce com a variabilidade das tarefas que eles desempenham; (ii) a ambiguidade das informações usadas nos departamentos cresce com a dificuldade da tarefa que desempenham; e (iii) a maneira com que as informações são usadas nos departamentos depende da variedade das tarefas que desempenham - quando a variabilidade é baixa as informações são usadas rápida e decisivamente, mas quando a variabilidade é alta, as informações são manipuladas lentamente. O Quadro 2 
O Impacto dos usos Interativo e Diagnóstico dos Orçamentos sobre a Eficácia das Equipes:

Retornando ao Básico

Gabriel Corrêa Teles Assis, Moacir Sancovschi, José Francisco Rezende

apresenta as características dos sistemas de informação que Daft e Macintosh (1981) identificaram nas quatro categorias de atividades definidas por Perrow (1967): sistemas concisos, sistemas superficiais, sistemas elaborados e sistemas difusos.

\begin{tabular}{|c|c|c|c|}
\hline & & \multicolumn{2}{|c|}{ Variedade da Tarefa } \\
\hline & & Baixa & Alta \\
\hline \multirow{2}{*}{ 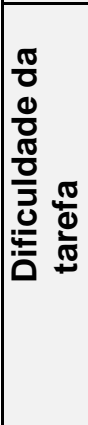 } & $\stackrel{\infty}{\frac{\pi}{4}}$ & $\begin{array}{l}\text { Artesanato } \\
\text { Sistema de Informação Superficial } \\
\text { Quantidade: Baixa } \\
\text { Ambiguidade: Alta } \\
\text { Uso: Rápido e Decisivo }\end{array}$ & $\begin{array}{l}\text { Tecnologia Não Rotineira } \\
\text { Sistema de Informação Difuso } \\
\text { Quantidade: Moderada } \\
\text { Ambiguidade: Alta } \\
\text { Uso: Manipulação Lenta }\end{array}$ \\
\hline & 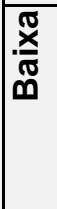 & $\begin{array}{l}\text { Tecnologia Rotineira } \\
\text { Sistema de Informação Conciso } \\
\text { Quantidade: Moderada } \\
\text { Ambiguidade: Baixa } \\
\text { Uso: Rápido e Decisivo }\end{array}$ & $\begin{array}{l}\text { Engenharia } \\
\text { Sistema de Informação Elaborado } \\
\text { Quantidade: Alta } \\
\text { Ambiguidade: Baixa } \\
\text { Uso: Manipulação Lenta }\end{array}$ \\
\hline
\end{tabular}

Quadro 2. Tecnologia dos Departamentos Usuários e Processamento de Informações Fonte: Adaptado de Daft e Macintosh (1981)

Abernethy e Stoelwinder (1991) entrevistaram gerentes de unidades de quatro hospitais sem fins lucrativos, e observaram que as unidades com melhor desempenho são aquelas que estão sob a responsabilidade de gerentes compromissados com preservação da adequada operação de suas unidades, e que percebem que os seus desempenhos são avaliados através de orçamentos usados por superiores esclarecidos (isto é, que reconhecem que o estilo com que os orçamentos devem ser usados depende da incerteza da atividade).

Chong (1996) entrevistou gerentes de companhias industriais e constatou que a utilização extensiva de sistemas de contabilidade de escopo amplo (que incluem informações externas, não financeiras e estimativas) em situações onde a incerteza da tarefa é alta contribuiu para a melhora do desempenho gerencial.

Sakka, Barki e Côté (2013) examinaram os fatores que explicam os estilos com que gerentes de projeto de desenvolvimento de sistemas de informação usam os sistemas de controle gerencial e o impacto que esses estilos têm no desempenho dos 
O Impacto dos usos Interativo e Diagnóstico dos Orçamentos sobre a Eficácia das Equipes:

Retornando ao Básico

Gabriel Corrêa Teles Assis, Moacir Sancovschi, José Francisco Rezende

projetos. Eles constataram, entre outras coisas, que o uso interativo dos controles melhorou o desempenho dos projetos onde o grau de complexidade e novidade era elevada, e reduziu o desempenho nos casos em que o grau de complexidade e novidade era baixo.

Grabner (2014) analisou a relação entre a dependência que as organizações têm da criatividade de seus funcionários para criar e produzir os produtos e serviços que comercializam e a intensidade com que elas usam pagamentos baseados em desempenho e avaliações subjetivas de desempenho nos seus projetos de sistemas de incentivos. Ela observou que quanto mais as empresas dependem da criatividade de seus funcionários mais combinam pagamentos por desempenho com avaliações subjetivas para incentivar seus funcionários, um modelo de incentivo compatível com sistemas de controle interativos (Simons, 1995).

Bloom, Eifert, Mahajan, McKenzie e Roberts (2011) realizaram um experimento de campo com indústrias têxteis da Índia, selecionadas aleatoriamente, para verificar a contribuição de três práticas gerenciais características dos sistemas de controle diagnósticos - (1) informações sobre o desempenho devem ser continuamente coletadas e analisadas, (2) metas de curto e de longo prazo integradas e desafiadoras devem ser estabelecidas, e (3) indivíduos e grupos de alto desempenho devem ser recompensados, e os que exibirem baixo desempenho devem ser treinados ou demitidos - para o desempenho de 28 fábricas dessas indústrias. Para este fim, eles proveram consultoria gratuita sobre as três práticas aos administradores de um subgrupo de fábricas, e compararam 0 desempenho dessas fábricas com 0 desempenho das fábricas de controle onde os administradores não receberam suporte dos consultores. Foram três os resultados que eles observaram. A adoção das práticas gerenciais (i) aumentou a produtividade em $11 \%$ através de ganhos de qualidade e de eficiência, e de redução dos investimentos em estoques; (ii) viabilizou um aumento na delegação de autoridade, e (iii) aumentou o uso de computadores nas fábricas.

Como as 28 fábricas que participaram do experimento de campo operavam 
O Impacto dos usos Interativo e Diagnóstico dos Orçamentos sobre a Eficácia das Equipes:

Retornando ao Básico

Gabriel Corrêa Teles Assis, Moacir Sancovschi, José Francisco Rezende

processos de produção contínua que funcionavam ininterruptamente, e boa parte dos problemas que elas tinham resultava de falta de disciplina, pode-se concluir que nelas havia todos os requisitos identificados por Hofstede (1981) e por Simons (1995) para usar, com sucesso, sistemas de controle diagnósticos.

Unger, Szczesny e Holderried (2019) examinaram empiricamente a influência da adoção de pagamentos por desempenho sobre a produtividade dos funcionários de unidades de hospitais responsáveis pela digitação de relatórios de alta dos pacientes (atividades rotineiras onde os incentivos são escassos). Eles observaram que, após a implantação dos pagamentos por desempenho, houve um aumento de aproximadamente $9,5 \%$ no número de páginas digitadas (isso representa, em um ano, um ganho de um mês de trabalho para os hospitais a uma fração do custo usual do serviço). Esse aumento se mostrou persistente, e não foram constatadas evidências de efeitos disfuncionais, nem de redução da qualidade do trabalho realizado.

Apesar de estas pesquisas terem sido feitas com base em protocolos distintos, é possível perceber que existem achados convergentes. Em atividades onde a incerteza é baixa há a percepção de que são adotados principalmente modos de coordenação e controle impessoais, entre eles estão os orçamentos. Nesses casos, os orçamentos refletem todas as atividades e/ou resultados associados ao desempenho da tarefa e se enquadram na categoria de sistemas concisos - trazem volume moderado de informações com substancial precisão e são usados de forma decisiva. Estas características são compatíveis com o uso diagnóstico dos orçamentos.

Em atividades onde a incerteza é alta há a percepção de que os principais modos de coordenação e controle são os modos pessoais e grupais. Nesses casos, os orçamentos, por não refletirem todas as atividades e/ou resultados associados ao desempenho da tarefa, se enquadram na categoria de sistemas difusos - trazem volume moderado de informações razoavelmente ambíguas que são manipuladas lenta e ponderadamente. Estas características são compatíveis com o uso interativo dos orçamentos. 
O Impacto dos usos Interativo e Diagnóstico dos Orçamentos sobre a Eficácia das Equipes:

Retornando ao Básico

Gabriel Corrêa Teles Assis, Moacir Sancovschi, José Francisco Rezende

\subsection{Modelo da Pesquisa}

Este trabalho tem como objetivos reafirmar, por meio de um estudo de caso, que, nas unidades das organizações onde a incerteza da tarefa é baixa, os orçamentos são usados de forma diagnóstica; e, nas unidades onde a incerteza da tarefa é alta, os orçamentos são usados de forma interativa; e que a adoção dos estilos de uso dos orçamentos adequados ao nível de incerteza da tarefa contribui para a eficácia coletiva percebida em equipes formais de trabalho e para a eficácia destas equipes. A Figura 1 apresenta o modelo adotado nesta pesquisa.

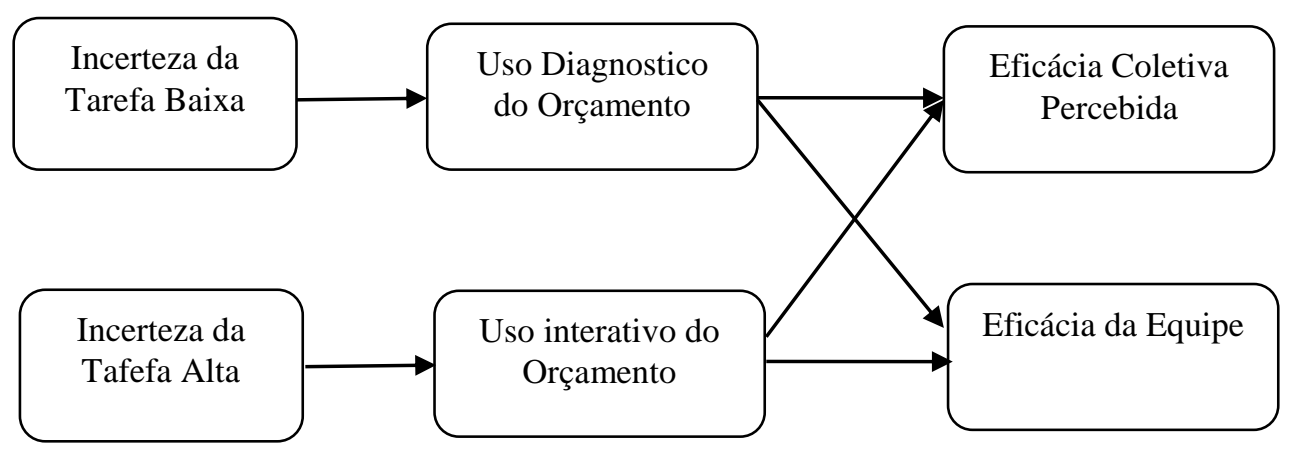

\section{Figura 1. Modelo da Pesquisa}

Fonte: Adaptação do Modelo Apresentado por Chong e Mahama (2014)

\section{PROCEDIMENTOS}

\subsection{Estratégia da Pesquisa}

Esta pesquisa foi realizada mediante um estudo de caso para investigar empiricamente as nuances das relações que há entre a incerteza das atividades desempenhadas em diferentes unidades organizacionais, a forma como os orçamentos são usados nessas em unidades, a eficácia coletiva percebida nas suas equipes de trabalho, e a eficácia dessas equipes. 
O Impacto dos usos Interativo e Diagnóstico dos Orçamentos sobre a Eficácia das Equipes:

Retornando ao Básico

Gabriel Corrêa Teles Assis, Moacir Sancovschi, José Francisco Rezende

\subsection{Unidades de Análise e Entrevistados}

Para garantir o alcance dos objetivos, foi necessário selecionar uma organização que possuísse unidades que desempenhassem atividades com níveis de incerteza distintos. Por uma questão de conveniência, a pesquisa foi aplicada no Instituto Brasileiro de Geografia e Estatística (IBGE).

No IBGE, as unidades analisadas foram a Coordenação de Geodésia e a Supervisão de Gravimetria, ambas subordinadas à Diretoria de Geociências.

Selecionadas estas unidades, a etapa seguinte foi identificar, em cada uma delas, um representante que possuísse experiência suficiente para descrever detalhadamente suas atividades e estivesse familiarizado com os processos de elaboração e utilização dos orçamentos, e com os principais controles de gestão existentes na unidade.

$\mathrm{Na}$ Coordenação de Geodésia, foi entrevistado o coordenador, que trabalha no IBGE há 32 anos e possui 10 anos (acumulados, porém não consecutivos) nessa função. No que diz respeito à Supervisão de Gravimetria da Gerência, o entrevistado foi o supervisor, que trabalha há 35 anos no órgão e possui os mesmos 35 anos no cargo atual.

Como os entrevistados são os funcionários de maior nível hierárquico nas duas unidades estudadas, é razoável considerar que eles atendem todos os requisitos necessários para fornecerem com competência e segurança as informações que este estudo necessita.

\subsection{Roteiro de entrevista}

As entrevistas seguiram roteiro semiestruturado, organizado em categorias que expressam os mesmos significados das variáveis da pesquisa Chong e Mahama (2014).

\subsubsection{Incerteza da Tarefa}

Diferentes autores têm usado definições operacionais distintas para a incerteza 
O Impacto dos usos Interativo e Diagnóstico dos Orçamentos sobre a Eficácia das Equipes:

Retornando ao Básico

Gabriel Corrêa Teles Assis, Moacir Sancovschi, José Francisco Rezende

da tarefa. Galbraith (1973) propôs que a incerteza da tarefa é função da diferença entre as informações possuídas na organização e as informações requeridas para a realização da tarefa. Ela é baixa quando a organização dispõe as informações requeridas antes que a tarefa seja realizada. Se assim for, a tarefa poderá ser préplanejada. Por outro lado, quando a organização não dispõe previamente das informações requeridas, o nível de incerteza é alto; e o conhecimento necessário ao desempenho da tarefa terá que ser obtido à medida que a tarefa é realizada.

Segundo Galbraith (1973), "a quantidade de informações requeridas para desempenhar uma atividade é função (i) da diversidade de outputs expressa pelo número de produtos, serviços ou clientes; (ii) do número de diferentes inputs empregados expresso pelo número de diferentes especialidades técnicas em um projeto, número de diferentes centros de máquinas, etc.; e (iii) do nível de dificuldade dos objetivos ou do desempenho expresso por algum critério de eficiência tal como percentagem de utilização das máquinas".

Para avaliar a incerteza da tarefa, o roteiro de entrevista adotou temas tratados nos questionários desenvolvidos e utilizados por Van de Ven, Delbecq e Koenig (1974). No entanto, não foi excluída possibilidade de que a definição operacional de Galbraith (1973) fosse utilizada, caso necessário para estimular os entrevistados a expressar as características das tarefas realizadas por suas equipes.

\subsubsection{Uso Interativo e Diagnóstico dos Orçamentos}

As fontes consultadas para a formulação das perguntas do roteiro que avaliaram os estilos com que os orçamentos eram usados foram: (i) as listas de características dos sistemas de controle diagnóstico e sistemas de controle interativo propostas por Simons (1995); (ii) as questões usadas nos questionários utilizados Chong e Mahama (2014).

\subsubsection{Eficácia Coletiva Percebida}

As perguntas usadas para avaliar a eficácia coletiva percebida em equipes 
O Impacto dos usos Interativo e Diagnóstico dos Orçamentos sobre a Eficácia das Equipes:

Retornando ao Básico

Gabriel Corrêa Teles Assis, Moacir Sancovschi, José Francisco Rezende

formais de trabalho foram fundamentadas em duas fontes principais: (i) as definições e o questionário de Chong e Mahama (2014); e (ii) a retrospectiva de Locke e Latham (2002).

\subsubsection{Eficácia da Equipe}

As questões de entrevista usadas para julgar a eficácia das equipes das duas unidades do IBGE foram baseadas, essencialmente, nas definições e no questionário de Chong e Mahama (2014).

\subsection{Fontes Documentais}

Para a adequada interpretação das informações obtidas nas entrevistas e para evitar tomar o tempo dos entrevistados além do necessário, foram consultados vários documentos oficiais da organização fornecidos aos autores pelos entrevistados.

\subsection{Limitações do Método}

Levando em conta unicamente os procedimentos metodológicos adotados, os achados desta pesquisa não poderiam ser generalizados. Porém, como esse trabalho foi concebido para constatar se resultados obtidos anteriormente também se manifestam no âmbito do IBGE, é razoável argumentar que ele contribui para a generalização analítica desses resultados (Yin, 2001).

Considerando que as informações usadas nesta pesquisa foram obtidas por meio de entrevistas com dois gerentes de unidades do IBGE e da leitura de documentos oficiais da organização, é importante reconhecer que as análises apresentadas a seguir e as conclusões alcançadas levam em conta percepções e opiniões pessoais dos entrevistados, e condições que deveriam prevalecer na organização se suas normas fossem completamente respeitadas, e não na realidade objetiva. 
O Impacto dos usos Interativo e Diagnóstico dos Orçamentos sobre a Eficácia das Equipes:

Retornando ao Básico

Gabriel Corrêa Teles Assis, Moacir Sancovschi, José Francisco Rezende

\section{O CASO ESTUDADO}

\subsection{O IBGE}

O Instituto Brasileiro de Geografia e Estatística (IBGE) é uma Fundação da Administração Pública Federal, atualmente subordinada ao Ministério da Economia. Sua missão institucional é "retratar o Brasil com informações necessárias ao conhecimento de sua realidade e ao exercício da cidadania".

O IBGE congrega quatro diretorias (Diretoria Executiva, Diretoria de Geociências, Diretoria de Pesquisas e Diretoria de Informática) e duas coordenaçõesgerais (Centro de Documentação e Disseminação de Informações e Escola Nacional de Ciências Estatísticas). Para que as atividades possam abranger toda a extensão do país, o IBGE possui ainda, entre outras divisões, 27 unidades estaduais, sendo 26 nas capitais e uma no Distrito Federal, e 581 agências de coleta de dados, nos principais municípios. Essas unidades planejam, coordenam, executam e controlam as atividades técnicas e administrativas do IBGE no limite de suas jurisdições.

\subsection{Diretoria de Geociências}

A Diretoria de Geociências é responsável por produzir e sistematizar as informações cartográficas, geodésicas, geográficas relativas aos recursos naturais e ao meio ambiente. Essas informações são imprescindíveis à análise, ao conhecimento, e à gestão do território nacional. Ela apoia as ações de planejamento do governo e permite que a sociedade tenha um retrato do país que é caracterizado pela diversidade de suas regiões.

A Diretoria de Geociências está organizada em Gerência de Documentação e Informação, Gerência de Geoinformações e Tecnologia, Gerência de Planejamento e Orçamento, Gerência de Levantamento Geodésico e Cartográfico, Coordenação de Cartografia, Coordenação de Estruturas Territoriais, Coordenação de Geodésia, Coordenação de Geografia e Coordenação de Recursos Naturais e Estudos Ambientais. 
O Impacto dos usos Interativo e Diagnóstico dos Orçamentos sobre a Eficácia das Equipes:

Retornando ao Básico

Gabriel Corrêa Teles Assis, Moacir Sancovschi, José Francisco Rezende

\subsubsection{Gerência de Planejamento e Orçamento}

A Gerência de Planejamento e Orçamento tem entre seus objetivos assessorar a Diretoria de Geociências no planejamento, na coordenação e na supervisão da utilização dos recursos orçamentários. Ela compila as demandas de recursos apresentadas por cada uma das coordenações e gerências dessa Diretoria.

Normalmente, os coordenadores e gerentes usam o orçamento vigente para elaborar o orçamento para o ano seguinte (orçamento incremental). De acordo com o coordenador da Coordenação de Geodésia, a Gerência de Planejamento e Orçamento define o orçamento que the caberá no ano seguinte e solicita que ele justifique qualitativamente onde os recursos serão usados. Ele, então, fundamenta suas decisões em documentos como os projetos estratégicos.

A compilação das demandas de todas as diretorias, coordenações gerais e unidades estaduais dá origem à proposta orçamentária geral do IBGE, que poderá ser incluída ou não na Lei Orçamentária Anual (LOA), a depender de sua aprovação pelo Congresso Nacional. A partir da aprovação da LOA, o Ministério da Economia libera os recursos que caberão ao IBGE para o ano corrente.

O controle dos recursos liberados é feito pela Diretoria Executiva. No âmbito da Diretoria de Geociências, cabe justamente à Gerência de Planejamento e Orçamento requisitar os recursos à Diretoria Executiva e distribuí-los entre as coordenações, conforme planejado anteriormente.

Esta Gerência acompanha as atividades realizadas pelas coordenações e gerencias ao longo do ano, por meio das informações que recebe sobre a progressão (em termos percentuais) dessas atividades. Dessa forma, caso as ações previstas no orçamento não sejam realizadas ou fiquem pendentes de conclusão, os coordenadores e gerentes são convidados a justificar o descumprimento dos planos e os atrasos ocorridos. 
O Impacto dos usos Interativo e Diagnóstico dos Orçamentos sobre a Eficácia das Equipes:

Retornando ao Básico

Gabriel Corrêa Teles Assis, Moacir Sancovschi, José Francisco Rezende

\subsubsection{Coordenação de Geodésia}

A Coordenação de Geodésia tem como objetivo fornecer uma infraestrutura geodésica de referência no Brasil, por meio de um conjunto de informações e serviços associados. Para o cumprimento deste objetivo, e com base nos requisitos legais atribuídos ao IBGE, essa coordenação administra o Sistema Geodésico Brasileiro, no que diz respeito ao seu estabelecimento, manutenção e acesso.

Este sistema é composto por estações geodésicas, que são pontos físicos espalhados pelo território brasileiro. Elas possuem componentes planimétricos (observações de direções horizontais - latitude e longitude), altimétricos (observações de altitude), maregráficos (observações do nível do mar) e gravimétricos (observações da aceleração da gravidade). As observações reunidas por esses componentes são fundamentais para atender as demandas de mapeamento, ordenamento da ocupação, implantação e manutenção de estruturas logísticas de transporte, de energia, saneamento e comunicação, monitoramento de mudanças climáticas e da elevação do nível médio do mar.

À Coordenação de Geodésia cabe, em última análise, o planejamento da manutenção estrutural das estações geodésicas, e dos levantamentos e medições feitas por seus componentes. Posteriormente, a coordenação processa os dados coletados e dissemina as análises resultantes nas plataformas digitais do IBGE.

A Coordenação é composta por oito gerências: Gerência de Planejamento, Gerência de Projetos, Gerência de Infraestrutura de Dados, Gerência de Redes de Referência Planialtimétricas, Gerência de Referência Vertical, Gerência de Manutenção das Redes Geodésicas, Gerência da Rede Gravimétrica e Desenvolvimento do Geóide e Gerência de Royalties do Petróleo. Ela é usuária dos serviços prestados pela Gerência de Levantamento Geodésico e Cartográfico.

No âmbito desta Coordenação, o orçamento é conhecido como Plano de Trabalho Anual. Esse Plano é elaborado em conjunto com a Gerência de Planejamento e Orçamento ao final de cada ano e nele estão previstas as atividades a serem executadas no exercício subsequente, assim como os desembolsos financeiros para 
O Impacto dos usos Interativo e Diagnóstico dos Orçamentos sobre a Eficácia das Equipes:

Retornando ao Básico

Gabriel Corrêa Teles Assis, Moacir Sancovschi, José Francisco Rezende

realizá-las.

\subsubsection{Incerteza da Tarefa na Coordenação de Geodésia}

Historicamente a Coordenação de Geodésia opera com escassez de recursos, visto que, por mais cuidadoso que seja o processo de elaboração do Plano de Trabalho Anual, ele é invariavelmente diminuído quando da aprovação da LOA. Assim, cabe argumentar que a redução no orçamento é relativamente previsível. Não obstante a previsibilidade da redução no volume de recursos solicitados no Plano de Trabalho Anual, ainda há a possibilidade de que eles sejam reduzidos ou contingenciados durante o exercício fiscal. Mas, as atividades a serem realizadas estão definidas e não podem ser alteradas. De acordo com o coordenador desta unidade, a questão de contingenciamento é antiga, sendo um instrumento frequentemente utilizado.

Este cenário enseja a ocorrência de eventos inesperados durante o cumprimento do Plano de Trabalho Anual que obrigam a frequente reprogramação das atividades da Coordenação, uma tarefa difícil que absorve a energia e o tempo de gerentes e funcionários.

A escassez de recursos, combinada com a razoável inflexibilidade das exigências técnicas para a realização das atividades, aumenta o grau de dificuldade dos objetivos da Coordenação, e consequentemente a quantidade de informações que ela requer para a realização das atividades, mas não constam das normas ou procedimentos institucionais.

Outro ponto importante a ser considerado é que, embora os modos de cálculo de altitudes e coordenadas estejam consolidados nos documentos técnicos, o coordenador afirma que ainda restam alguns "desafios", tais como demandas excepcionais, que exigem maior esforço e tempo de preparo.

Os problemas causados por estes casos excepcionais, segundo o coordenador, são atenuados com treinamentos e a capacitação dos funcionários. Para isso, são convidados professores universitários ou outros especialistas das disciplinas relacionadas ao trabalho, já que eles possuem maior experiência que os servidores da 
O Impacto dos usos Interativo e Diagnóstico dos Orçamentos sobre a Eficácia das Equipes:

Retornando ao Básico

Gabriel Corrêa Teles Assis, Moacir Sancovschi, José Francisco Rezende

coordenação, que muitas vezes ainda não tiveram a oportunidade de se especializar, através de cursos de pós-graduação, por exemplo.

Finalmente, os recursos humanos são também, segundo o coordenador, uma fonte de dificuldade e excepcionalidade. Como no âmbito da Coordenação de Geodésia o trabalho depende muito da ação humana, e consequentemente, do bem-estar físico e mental dos servidores, isso se torna uma preocupação que reside principalmente no fato de o coordenador não ter gestão sobre eventuais problemas de natureza particular do servidor.

Enfim, a escassez endêmica de recursos, combinada com a necessidade de atender a demandas sistemáticas e eventuais que exigem a utilização de técnicas e equipamentos sofisticados e de rotinas que são razoavelmente rígidas, e requerem a disponibilidade de funcionários qualificados em permanente processo de atualização, aumentam a quantidade de informações necessárias para o desempenho das atividades, e que não estão disponíveis na Coordenação. Isso impõe que informações sejam processadas à medida que o trabalho evolui para garantir que os objetivos sejam alcançados sem que haja o comprometimento dos padrões de qualidade dos serviços realizados, como previsto no modelo de Galbraith (1973).

\subsubsection{Orçamento na Coordenação de Geodésia}

O Plano de Trabalho Anual da Coordenação de Geodésia é um plano de curto prazo que está inserido no contexto do Plano Estratégico do IBGE que tem periodicidade decenal. Ele é elaborado ao final de cada ano, devendo ser entregue até o mês de dezembro à Gerência de Planejamento e Orçamento e, posteriormente, à Diretoria Executiva. Além do coordenador da Coordenação de Geodésia e do gerente da Gerência de Planejamento e Orçamento, participam da elaboração do orçamento os representantes das gerências desta Coordenação, que por sua vez reúnem informações a partir do contato com os setores executantes dos levantamentos em campo. Verifica-se, portanto, a participação de todos os níveis hierárquicos nas definições do Plano de Trabalho Anual. 
O Impacto dos usos Interativo e Diagnóstico dos Orçamentos sobre a Eficácia das Equipes:

Retornando ao Básico

Gabriel Corrêa Teles Assis, Moacir Sancovschi, José Francisco Rezende

Os dados e premissas que usados no Planejamento Estratégico e nos orçamentos são permanentemente questionados e revistos. De acordo com o Relatório de Gestão 2018, o processo de Planejamento Estratégico e seu(s) Plano resultante(s) são dinâmicos porque são impactados por alterações nos ambientes interno e externo, impondo mudanças de percurso à medida que são implementados (IBGE, 2018).

O Plano de Trabalho Anual tem um acompanhamento físico e financeiro consolidado mensalmente pela Coordenação de Geodésia. Isto significa que as atividades previstas para um mês "M" devem em regra ser confirmadas até a primeira quinzena do mês "M-1". No caso de realização de atividades incluídas no planejamento, canceladas, reprogramadas ou de campanhas emergenciais não planejadas, a coordenação analisa se a reprogramação ou inclusão de atividades pode prejudicar a execução física de outros projetos. Segundo o coordenador, o Plano de Trabalho Anual é um "pontapé inicial", e ao longo dos meses revisões são frequentes e necessárias.

Isto sugere que desvios em relação ao orçamento inicial são admitidos, pois o Plano de Trabalho Anual configura apenas o ponto de partida para as discussões que permitirão a atuação de modo eficiente. Geralmente, a Coordenação de Geodésia não é avaliada pelo cumprimento integral do planejamento inicial, conforme afirmou seu coordenador. Segundo ele, cumprir o orçamento é uma atividade que requer "jogo de cintura". Resta evidenciado que a avaliação do cumprimento do Plano de Trabalho Anual está mais baseada no reconhecimento dos esforços realizados do que nos resultados alcançados.

Em resumo, o Plano de Trabalho Anual na Coordenação de Geodésia formaliza o envolvimento dos gerentes nas atividades das equipes por meio de reuniões; estimula o debate e incentiva o contínuo desafio dos pressupostos fundamentais e planos de ação das equipes; e facilita a construção de um consenso entre os membros da equipe, direcionando a atenção para questões comuns e fatores críticos de sucesso. Isto é, o Plano de Trabalho Anual é usado de forma interativa, conforme a definição de Simons (1995). 
O Impacto dos usos Interativo e Diagnóstico dos Orçamentos sobre a Eficácia das Equipes:

Retornando ao Básico

Gabriel Corrêa Teles Assis, Moacir Sancovschi, José Francisco Rezende

\subsubsection{Eficácia da Equipe e Eficácia Coletiva Percebida na Coordenação de Geodésia}

O Relatório de Gestão 2018 do IBGE apresenta números relativos à produtividade da Coordenação de Geodésia. Ele mostra que, em relação ao conjunto de estações que consolidam as observações de altitude do Sistema Geodésico Brasileiro por meio de medições de nivelamento geométrico de alta precisão, foram colocadas à disposição 68 mil novas altitudes físicas, resultantes do Reajustamento Altimétrico 2018.

Ainda conforme este relatório, a Rede Brasileira de Monitoramento Contínuo, que monitora as variações posicionais que ocorrem na superfície da Terra ao longo do tempo, foi expandida em 10\% em relação ao ano de 2017. Atualmente, essa rede está operando 152 estações. No âmbito de operação dessa rede, foram colocados à disposição 49.235 arquivos diários, acumulando um aumento de $36,76 \%$ em relação ao ano anterior. A obtenção de tais marcas foi possível através da minimização da taxa de perda de dados das estações e da expansão da rede. Com relação à Rede Maregráfica Permanente para Geodésia, que realiza o monitoramento do nível do mar em diversos pontos da costa brasileira, um conjunto de 1.400 arquivos foi publicado no portal do IBGE, segundo informa o mesmo Relatório de Gestão 2018.

De modo geral, o coordenador da Coordenação de Geodésia parece estar satisfeito com a eficácia da equipe. Para ele, a equipe tem realizado um trabalho competente, e o processo de crítica constante à medida que o trabalho é desenvolvido é fundamental para o aprimoramento desta competência.

Esta satisfação diz respeito tanto à quantidade quanto à qualidade do serviço. No tocante à pontualidade no cumprimento dos cronogramas, o coordenador afirma que está satisfeito "na maioria dos casos".

Há, por parte do coordenador, a confiança na capacidade da sua equipe desempenhar satisfatoriamente as atividades que lhe cabem, porém com uma ressalva: os servidores mais novos, os quais têm no IBGE a sua primeira experiência profissional. Nesses casos, ele reconhece que é preciso um tempo para consolidar o conhecimento 
O Impacto dos usos Interativo e Diagnóstico dos Orçamentos sobre a Eficácia das Equipes:

Retornando ao Básico

Gabriel Corrêa Teles Assis, Moacir Sancovschi, José Francisco Rezende

e um acompanhamento mais cuidadoso. O coordenador também acredita que, com o apoio de outras esferas da instituição, a equipe é capaz de lidar de forma eficaz com problemas inesperados.

Em geral, as declarações do coordenador a respeito de como as atividades são realizadas e a confiança que ele expressa na capacidade de seus subordinados desempenhá-las com sucesso levam a crer que ele está satisfeito e aprova o desempenho das equipes da Coordenação de Geodésia. Neste sentido, é razoável supor que, se o coordenador respondesse aos questionários de Chong e Mahama (2014), ele acusaria que a eficácia coletiva percebida e a eficácia das equipes são altas nesta Coordenação.

\subsection{Supervisão de Gravimetria}

A Gerência de Levantamento Geodésico e Cartográfico pode ser entendida como uma prestadora de serviços no âmbito do IBGE. Cabe a ela planejar e coordenar as atividades de levantamentos de campo para a Coordenação de Geodésia, a Coordenação de Cartografia e a Coordenação de Estruturas Territoriais.

Os levantamentos de campo envolvem a implantação, a medição e a verificação das condições físicas das estações geodésicas do Sistema Geodésico Brasileiro; a medição de pontos de referência necessários ao registro de imagens aéreas ou via satélite, e a posterior correção destas imagens, devido a distorções geométricas; a avaliação da posição de documentos cartográficos; a coleta e certificação de nomes geográficos; e a implantação e medição de estruturas para a manutenção do conjunto de informações territoriais brasileira, com sua divisão político-administrativa.

A Figura 2 apresenta a estrutura da Gerência de Levantamento Geodésico e Cartográfico, que se subdivide em Planejamento e Apoio Administrativo, Supervisão de Planimetria e Supervisão de Gravimetria. 


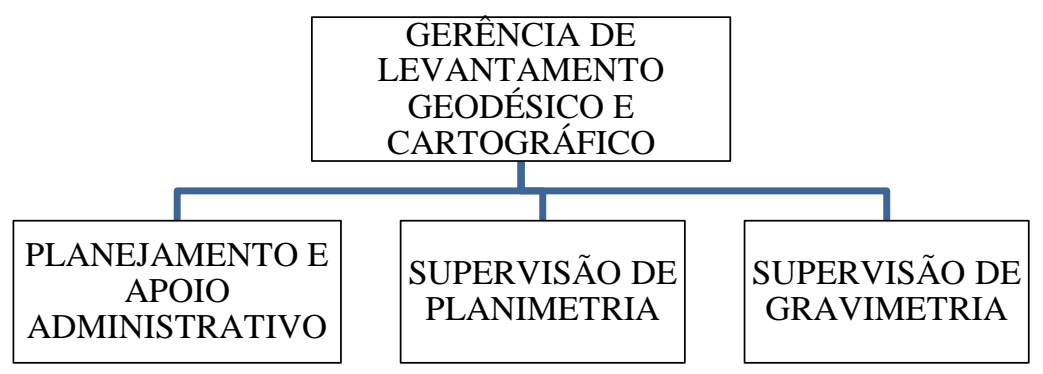

Figura 2. A Gerência de Levantamento Geodésico e Cartográfico

Fonte: Adaptado IBGE (2019)

A supervisão de Gravimetria é a equipe responsável pelos levantamentos de campo relacionados ao conjunto de estações geodésicas denominadas estações gravimétricas, que contém informações sobre a aceleração da gravidade e visam recobrir, no território brasileiro, os grandes vazios destas informações. A informação gravimétrica é importante especialmente para a área da Geodésia (IBGE, 2019).

De modo geral, as atividades a serem realizadas em campo pela Supervisão de Gravimetria estão previstas no Plano de Trabalho Anual e o trabalho das equipes responsáveis pelos procedimentos se baseia neste documento. A esta altura, os recursos financeiros previstos no Plano de Trabalho Anual já foram alocados, principalmente sob a forma de diárias, passagens, compra de instrumentos e demais insumos necessários à execução dos serviços. No entanto, é ainda disponibilizada certa quantia de recursos aos servidores, para suprir eventuais gastos durante a execução do trabalho no campo.

\subsection{Incerteza da Tarefa na Supervisão de Gravimetria}

As atividades realizadas pela Supervisão de Gravimetria podem ser consideradas repetitivas, e, são poucos, os eventos imprevistos que ocorrem enquanto elas são realizadas.

De acordo com o entrevistado, quando eventualmente surge alguma dúvida na 
O Impacto dos usos Interativo e Diagnóstico dos Orçamentos sobre a Eficácia das Equipes:

Retornando ao Básico

Gabriel Corrêa Teles Assis, Moacir Sancovschi, José Francisco Rezende

execução do trabalho, ela normalmente é sanada por meio de consulta ao manual do equipamento GPS que se está operando. "O manual dá todo o suporte. Dificilmente nós ficamos sem saber o que fazer", disse o supervisor. "Nas raras vezes em que a equipe se depara com imprevistos e não sabe como resolver, seu chefe consulta as gerências técnicas responsáveis por planejar as atividades".

Apesar de existir a possibilidade de a Supervisão de Gravimetria prestar serviços para outras coordenações e até diretorias fora de seu escopo, tal solicitação geralmente é para manusear um equipamento GPS. Portanto, nesses casos, o trabalho é similar ao que os técnicos já estão habituados a fazer.

Foi verificado que a previsibilidade da aplicação dos recursos financeiros concedidos para eventuais despesas reflete a própria previsibilidade da natureza das tarefas que competem às equipes nos trabalhos de campo. À medida que as atividades se repetem, repetem-se também as necessidades que justificam o uso da importância financeira disponível. Ademais, como os servidores que detêm os recursos não podem dispor deles livremente, são poucas as decisões que podem ser tomadas em sua aplicação.

Para Galbraith (1973), a incerteza da tarefa é baixa quando a organização dispõe as informações requeridas antes que a tarefa seja realizada. Nestes casos, a tarefa poderá ser pré- planejada. Como na Supervisão de Gravimetria todas as informações requeridas estão geralmente disponíveis, pode-se argumentar que a incerteza da tarefa é baixa nesta unidade.

Neste ponto, fica patente que as Coordenações que usam os serviços desta Supervisão absorvem grande parte da incerteza do processo, permitindo que esta última tenha condições de pré-planejar suas atividades. Compete à Supervisão de Gravimetria realizar, com zelo e disciplina, as atividades especificadas pelas Coordenações que as requisitaram.

\subsection{Orçamento na Supervisão de Gravimetria}

Diferentemente do que ocorre na Coordenação de Geodésia, o orçamento na 
O Impacto dos usos Interativo e Diagnóstico dos Orçamentos sobre a Eficácia das Equipes:

Retornando ao Básico

Gabriel Corrêa Teles Assis, Moacir Sancovschi, José Francisco Rezende

Supervisão de Gravimetria não consiste em um único documento. Ele está desmembrado, possuindo duas dimensões: uma física e outra financeira.

A dimensão física refere-se ao Plano de Trabalho Anual onde se encontra o planejamento das atividades que serão desempenhadas pela equipe em campo. Esse documento norteia a equipe na execução dos serviços que cabem à Supervisão. Após realizar as atividades previstas no Plano de Trabalho Anual, a equipe faz análises preliminares dos dados levantados e as submetem às unidades demandantes.

Por outro lado, a dimensão financeira apresenta os recursos distribuídos para apoio durante a realização das atividades da Supervisão de Gravimetria. Este instrumento é denominado suprimento de fundos.

O suprimento de fundos é um regime de adiantamento de valores a um servidor (em espécie ou na forma de cartão de crédito) para futura prestação de contas, utilizado quando não se pode submeter um gasto ao processo normal de licitação. Assim, esse regime serve para atender despesas eventuais, de caráter sigiloso ou de pequeno vulto (MCASP, 2019).

Como exemplo de finalidade dos suprimentos de fundos durante as campanhas da Supervisão de Gravimetria, é possível citar a aquisição de combustível para abastecimento de veículos, compra de materiais de consumo ou ferramentas necessários à execução da atividade, contratação de mão-de-obra especializada para um fim particular, etc.

A subdivisão de Apoio e Planejamento e a Supervisão de Gravimetria preparam o orçamento para determinada campanha, com base na distância do deslocamento, no número de dias que ela durará e nas características da atividade a ser desempenhada. Para as atividades relacionadas à geodésia, esta programação precisa, na medida do possível, estar de acordo com o que já foi previsto pela Coordenação de Geodésia quando da elaboração do Plano de Trabalho Anual.

Existe uma comunicação formal ao servidor no ato de concessão de suprimento de fundos, informando os limites financeiros que devem ser respeitados, inclusive discriminados por tipo de gasto (ex: material de consumo, contratação de 
O Impacto dos usos Interativo e Diagnóstico dos Orçamentos sobre a Eficácia das Equipes:

Retornando ao Básico

Gabriel Corrêa Teles Assis, Moacir Sancovschi, José Francisco Rezende

pessoa física, contratação de pessoa jurídica, transporte, etc.). Após a aplicação dos recursos, os servidores devem prestar contas à Diretoria Executiva, mediante a apresentação de todas as notas fiscais referentes aos gastos.

Como nesta etapa não cabe mais discutir realocações, os limites do suprimento de fundos devem ser obrigatoriamente respeitados. A análise da prestação de contas consiste principalmente em comparar os valores gastos com aqueles planejados. Caso haja gastos além daquele autorizado, salvo exceções, os servidores são notificados a devolver os valores ultrapassados. As exceções mencionadas dizem respeito a casos muito específicos, em que o diretor da Diretoria de Geociências autoriza um gasto acima do planejado por julgar que ele seria fundamental para a execução da atividade.

Em síntese, na Supervisão de Gravimetria, o Plano de Trabalho Anual e o suprimento de fundos definem as atividades que deverão ser realizadas pela equipe e os recursos que deverão ser usados por ela, os relatórios periódicos são usados para avaliar o progresso da equipe no cumprimento dos objetivos físicos e financeiros, e decisões são tomadas quando há desvios negativos em relação aos planos. Isto é, o Plano de Trabalho Anual e suprimento de fundos são usados de forma diagnóstica, conforme a definição de Simons (1995).

\subsection{Eficácia da Equipe e Eficácia Coletiva Percebida na Supervisão de Gravimetria}

Alguns dados relativos às campanhas realizadas pelo IBGE constam no Relatório de Gestão 2018. Nesse ano foi concluído o reestabelecimento das redes geodésicas do estado do Amapá totalizando oitocentos e quarenta novos marcos geodésicos. Além disso, houve a atualização de informações referentes à 2019 estações geodésicas no Banco de Dados Geodésicos.

Destaca-se também a inclusão de 84 novas estações de GPS de alta precisão sobre o território brasileiro, a homologação de 38 novas estações de GPS oriundas de contribuições de entidades externas, a medição e carga no banco de dados 
O Impacto dos usos Interativo e Diagnóstico dos Orçamentos sobre a Eficácia das Equipes:

Retornando ao Básico

Gabriel Corrêa Teles Assis, Moacir Sancovschi, José Francisco Rezende

geodésicos de 3.789 novas estações gravimétricas (com incremento de $26 \%$ de produtividade prevista em 2018, decorrente da implantação de novas metodologias de aquisição de dados) e a visita a aproximadamente 1.200 marcos geodésicos.

No que diz respeito aos recursos aplicados através de suprimento de fundos durante as campanhas da Diretoria de Geociências no ano de 2018, não houve nenhum processo impugnado durante este exercício. Isso significa que todo o planejamento da Diretoria de Geociências para a aplicação de suprimento de fundos foi rigorosamente atendido.

$\mathrm{Na}$ percepção do supervisor de Gravimetria da Gerência de Levantamento Geodésico e Cartográfico, a sua equipe é eficaz. Ele afirma estar satisfeito com os resultados apresentados tanto em termos de quantidade quanto, principalmente, em termos de qualidade. A pontualidade das entregas também é destacada pelo entrevistado, havendo casos em que os trabalhos são concluídos antes dos prazos previstos. Ressalvados os casos de eventuais imprevistos, as equipes, segundo o entrevistado, são capazes de realizar todas as atividades empregando os recursos financeiros e materiais disponíveis.

A confiança na equipe, de acordo com o supervisor, é condição essencial para a execução do trabalho. No entanto, ele reconhece que a experiência e que a responsabilidade individual são fatores determinantes para a confiança na equipe.

A Supervisão de Gravimetria lida com as atividades difíceis por meio de diálogos com o seu supervisor. Desta forma, os membros da equipe têm a liberdade de procurar o supervisor para dirimir suas dúvidas.

Em geral, as declarações do supervisor a respeito de como as atividades são realizadas, e a confiança que ele expressa na capacidade de seus subordinados desempenhá-las com sucesso levam a crer que ele está satisfeito e aprova o desempenho da Supervisão de Gravimetria. Nesse sentido, pode-se supor que, se o supervisor respondesse aos questionários de Chong e Mahama (2014), ele acusaria que a eficácia coletiva percebida e a eficácia das equipes são altas nesta Gerência. 
O Impacto dos usos Interativo e Diagnóstico dos Orçamentos sobre a Eficácia das Equipes:

Retornando ao Básico

Gabriel Corrêa Teles Assis, Moacir Sancovschi, José Francisco Rezende

\section{CONSIDERAÇÕES FINAIS}

Este trabalho teve como objetivos reafirmar, por meio de um estudo de caso, que nas unidades das organizações onde a incerteza da tarefa é baixa, os orçamentos são usados de forma diagnóstica; e, nas unidades onde a incerteza da tarefa é alta, os orçamentos são usados de forma interativa; e que a adoção dos estilos de uso dos orçamentos adequados ao nível de incerteza da tarefa contribui para a eficácia coletiva percebida em equipes formais de trabalho e para a eficácia destas equipes.

Para este propósito, foram analisadas as atividades desempenhadas pelas equipes da Coordenação de Geodésia e da Supervisão de Gravimetria, os estilos com que os orçamentos são usados em cada uma dessas unidades, a eficácia coletiva percebida pelas equipes de trabalho em cada uma dessas unidades e a eficácia dessas equipes.

Esta pesquisa identificou e descreveu duas configurações. A primeira está na Coordenação de Geodésia: realiza tarefas com elevado nível de incerteza, nela o orçamento é usado de forma interativa, e o seu coordenador expressou a sensação de que a equipe é qualificada e competente, e eficaz, levando-o a assumir que a eficácia da equipe e a eficácia coletiva percebida são ambas satisfatórias no âmbito dessa unidade.

A segunda configuração está na Supervisão de Gravimetria: sempenha tarefas com baixo nível de incerteza, nela o orçamento é usado de forma diagnóstica e o seu supervisor expressou a sensação de que os resultados são positivos e de que confia na capacidade de sua equipe, levando-o a assumir que a eficácia da equipe e a eficácia coletiva percebida são ambas satisfatórias no âmbito dessa unidade.

Cumpre ressaltar que, a despeito de os resultados deste estudo não serem generalizáveis, eles estão alinhados com os resultados de pesquisas anteriores, realizadas em diferentes ocasiões, setores e locais de operação. Sendo assim, eles reafirmam e reforçam resultados anteriores mostrando que eles são analiticamente generalizáveis. 
O Impacto dos usos Interativo e Diagnóstico dos Orçamentos sobre a Eficácia das Equipes:

Retornando ao Básico

Gabriel Corrêa Teles Assis, Moacir Sancovschi, José Francisco Rezende

Embora o coordenador da Coordenação de Geodésia não tenha feito qualquer menção ao uso diagnóstico de orçamentos nessa Coordenação, isso não significa que esse estilo não fosse usado nessa unidade. Há de se considerar que nessa Coordenação o uso diagnóstico somente se aplica ao controle de atividades secundárias com baixo nível de incerteza. Daí o coordenador ter dado amplo destaque ao uso interativo dos orçamentos por considerá-lo mais saliente e relevante.

A principal contribuição deste trabalho reside na indicação de que a análise e o projeto de sistemas de controle não podem prescindir de uma avaliação do nível de incerteza da tarefa nos locais onde eles são ou serão aplicados.

Do ponto de vista prático, o caso descrito nesta pesquisa sublinha a importância de se reconhecer que os orçamentos são relatórios financeiros que não indicam a seus como devem utilizá-los. Logo, seus usuários precisam ser lembrados que de que o adequado uso dos orçamentos deve ser precedido por uma avaliação do nível de incerteza das atividades.

\section{REFERÊNCIAS}

Abernethy, M. A., \& Stoelwinder, J. U. (1991). Budget use, task uncertainty, system goal orientation and subunit performance: a test of the "fit" hypothesis in not-for-profit hospitals. Accounting, Organizations and Society, 16, 105-120.

Anthony, R. N. (1974). Framework for analysis. In: De Coster, Ramanathan, K. V., \& Sundem, G. L. Accounting for managerial decision making.

Atkinson, A. A., Kaplan, R. S., Matsumura, E. M., \& Young, S. M (2015). Contabilidade gerencial: informação para a tomada de decisão e execução da estratégia.

Bloom, N., Eifert, B., Mahajan, A., Mckenzie, D., \& Roberts, J. (2011). Does management matter? Evidence from India. NBER Working Paper, 16658, 2011.

Chong, V., K. (1996). Management Accounting Systems, Task Uncertainty and Managerial Performance: a Research Note. Accounting, Organizations and Society, 21 (5), 415- 421.

Chong, K. M., \& Mahama, H. (2014). The impact of interactive and diagnostic uses of 
O Impacto dos usos Interativo e Diagnóstico dos Orçamentos sobre a Eficácia das Equipes:

Retornando ao Básico

Gabriel Corrêa Teles Assis, Moacir Sancovschi, José Francisco Rezende

budgets on team effectiveness. Management Accounting Research, 25 (3), 206-222.

Daft, R. L., \& Macintosh, N. B. (1981). A Tentative Exploration into the Amount and Equivocality of Information Processing in Organizational Work Units. Administrative Science Quartely, 26 (2), 207-224.

Dermer, J. (1977). Management planning and control systems: advanced concepts and cases. Irwin, Homewood.

Galbraith, J. R. (1973). Designing Complex Organizations. Boston, Addison-Wesley.

Garrison, R. H., Noreen, E. W., \& Brewer, P. C (2013). Contabilidade Gerencial. Porto Alegre, McGraw Hill Bookman.

Grabner, I. (2014). Incentive System Design in Creativity- Dependent Firms. The Accounting Review, $89(5), 1729-1750$.

Hofstede, G. (1981). Management control of public and not-for-profit activities. Accounting, Organizations and Society, 6 (3), 193-211.

Hopwood, A. G. (1972). An Empirical Study of the Role of Accounting Data in Performance Evaluation. Journal of Accounting Research, 10, 156-182.

Hoorngren, C. T., Datar, S. M., \& Rajan, M. V. (2015). Cost accounting: a managerial emphasis. Englewood Cliffs, Pearson Prentice Hall.

Instituto Brasileiro de Geografia e Estatística. Relatório de Gestão. Recuperado de: https://www.ibge.gov.br/np_download/novoportal/documentos _institucionais/Relatorio_gestao. Acesso em: 11/jul./2019.

Instituto Brasileiro de Geografia e Estatística. Sistema Geodésico Brasileiro - Rede Gravimétrica. Recuperado de: https://ww2.ibge.gov.br/home/geociencias/geodesia/gravimetrica.shtm, Acesso em: 11/jul./2019.

Landau, M., \& Stout Jr, R. (1979). To manage is not to control: or the folly of type II errors. Public Administration Review, 148-156.

Merchant, K. A. (1985). Control in business organizations. Ballinger Publishing Company.

Merchant, K. A., \& Van der Stede, W. A. (2012). Management control systems: performance measurement, evaluation and incentives. Prentice Hall. 
O Impacto dos usos Interativo e Diagnóstico dos Orçamentos sobre a Eficácia das Equipes:

Retornando ao Básico

Gabriel Corrêa Teles Assis, Moacir Sancovschi, José Francisco Rezende

Mintzberg, H. (1987). The strategy concept i: five ps for strategy. California management Review, 30 (1), 11-24.

Otley, D. T. (1978). Budget Use and Managerial Performance. Journal of Accounting Research, 16 (1), 122-149.

Ouchi, W. G. (1979). A conceptual framework for the design of organizational control mechanisms. Management Science, 25 (9), 833-849.

Perrow, C. A. (1967). Framework for the Comparative Analysis of Organizations. American Sociological Review, 32 (2), 194-208.

Sakka, O., Barki, H., \& Côté, L. (2013). Interactive and diagnostic uses of management control systems in is projects: antecedents and their impact on performance. Information \& Management, 50, 265-274.

Samuelson, P. (1973). Economics. Mcgraw-Hill Kogakusha.

Secretaria do Tesouro Nacional. Manual de Contabilidade Aplicada ao Setor Público. Brasília, 2019. Recuperado de: https://www.tesourotransparente.gov.br/publicacoes/manual-de-contabilidadeaplicada-ao-setor-publico-mcasp/2019/26. Acesso em: 21/jan./2019.

Seijts, G. H., \& Latham, G. P. (2012). Knowing when to set learning versus performance goals. Organization Dynamics, 41 (1), 1-6.

Simons, R. (1995). Levers of Control: How Managers use Innovative Control Systems to Drive Strategic Renewal. Boston. Harvard Business School.

Van de Ven, A. H., Delbecq, A. L. A., \& Koenig JR., R. (1976). Determinants of Coordination Modes within Organizations. American Sociological Review, 41, 322338.

Yin, R. K. (2001). Estudo de Caso: Planejamento e Métodos. Porto Alegre, Bookman.

Data de Submissão: 15/10/2020

Data de Aceite: 15/12/2020 